\title{
High-Accuracy Tracking Using Ultrawideband Signals for Enhanced Safety of Cyclists
}

\author{
Davide Dardari, ${ }^{1}$ Nicoló Decarli, ${ }^{1}$ Anna Guerra, ${ }^{1}$ Ashraf Al-Rimawi, ${ }^{1}$ \\ Víctor Marín Puchades, ${ }^{2}$ Gabriele Prati, ${ }^{2}$ Marco De Angelis, ${ }^{2}$ Federico Fraboni, ${ }^{2}$ \\ and Luca Pietrantoni ${ }^{2}$ \\ ${ }^{1}$ Department of Electrical, Electronic and Information Engineering "Guglielmo Marconi", The University of Bologna, Bologna, Italy \\ ${ }^{2}$ Department of Psychology, The University of Bologna, Bologna, Italy
}

Correspondence should be addressed to Davide Dardari; davide.dardari@unibo.it

Received 18 November 2016; Revised 30 January 2017; Accepted 9 February 2017; Published 15 March 2017

Academic Editor: Cristiano Silva

Copyright (C) 2017 Davide Dardari et al. This is an open access article distributed under the Creative Commons Attribution License, which permits unrestricted use, distribution, and reproduction in any medium, provided the original work is properly cited.

In this paper, an ultrawideband localization system to improve the cyclists' safety is presented. The architectural solutions proposed consist of tags placed on bikes, whose positions have to be estimated, and anchors, acting as reference nodes, located at intersections and/or on vehicles. The peculiarities of the localization system in terms of accuracy and cost enable its adoption with enhanced risk assessment units situated on the infrastructure/vehicle, depending on the architecture chosen, as well as real-time warning to the road users. Experimental results reveal that the localization error, in both static and dynamic conditions, is below $50 \mathrm{~cm}$ in most of the cases.

\section{Introduction}

In recent years, vulnerable road users (VRUs) injuries and mortality have rapidly grown due to the increased road traffic: around 2000 people riding bicycle are killed every year in traffic accidents in EU countries [1] and about $30 \%$ of cyclist fatalities take place at junctions $[1,2]$. In this scenario, it becomes urgent to develop solutions in order to both encourage people to cycle and make cycling safer at the same time. As regards the last point, increasing road users' awareness of cyclists' presence in the surrounding becomes an essential issue along with the possibility of warning cyclists during dangerous maneuvers of cars and heavy goods vehicles (HGVs).

Today several technologies are available and could be exploited to detect dangerous situations for cyclists. In order to choose the proper one, we propose a multidisciplinary approach based on a deep analysis of the most dangerous scenarios and situations. In this context, the starting point is a behavioral investigation which takes a central role in establishing the users' acceptance, to enhance the cyclists' comfort together with their safety and to put the final user at the center of all the investigation and the ultimate aim of the technologies that will be developed. For these reasons, the technological requirements for such application must account for several and heterogeneous aspects in order to make it effective, reliable, and user-friendly and with low-cost.

To this end, in this paper we first provide an analysis of accidents statistics involving cyclists. Second, a survey of existing technologies, with a particular emphasis on their pros and cons, is undertaken. Starting from the previous outcomes, we propose a new detection architecture providing high-accuracy localization and tracking of road users based on the ultrawideband (UWB) technology [3]. UWB, in its IEEE802.15.4a standard implementation [4], has already proved to be the best candidate in achieving high localization accuracy at low cost, thanks to its high temporal resolution and the ability to resolve multipath and to coexist with other wireless technologies [5-7]. We experimentally characterized the performance of the implemented UWB-based tracking system by means of indoor and outdoor measurements campaigns, as detailed in the numerical results.

The availability of accurate real-time tracking of road users opens the door to the introduction of advanced risk 
assessment (RA) units, both on vehicles and on the infrastructure (on-site), capable of predicting critical situations and providing a suitable feedback to the road user through ad hoc human machine interfaces (HMIs). Moreover, it also enables the possibility of offering additional services to cyclists, such as enhancing the functionality of green waves by accounting for the amount of people approaching the traffic light. These applications are currently being investigated in the Europefunded project XCycle (http://www.xcycle-h2020.eu).

The rest of the paper is organized as follows: Section 2 presents an analysis of accident statistics involving cyclists; Section 3 reports a summary of the available technologies for detection of cyclists; Section 4 presents the considered architecture for enhanced safety of cyclists; Section 5 describes the localization method considered exploiting UWB signals; Section 6 shows some experimental results characterizing the system performance; finally, Section 7 concludes the paper.

\section{Analysis of Accident Statistics Involving Cyclists}

2.1. Analysis of Accidents Reports. In 2012, 2143 bicyclists died in road accidents in Europe [1]. Although the number of cycling fatalities did not increase from 2004 to 2013, the proportion of cyclist fatalities with reference to overall road fatalities has a growing trend [1]. The majority (52\%) of cyclist deaths on the European roads between 2011 and 2013 had passenger cars as opponent vehicles, whereas the $24 \%$ involved goods vehicles or buses/coaches [8]. Cyclist accidents involving HGVs have shown to be more fatal given that HGVs are involved in $12 \%$ of cyclist accidents (data from 2005 to 2010) [2]. According to the data provided by the Italian National Institute of Statistics [9], 575.093 road accidents occurred in the period ranging from 2011 to 2013, on the Italian road network. Of these, 49.621 road accidents involved at least one injured or killed cyclist. The number of bicycle fatalities during this period was 823 (1.7\% of roads accidents involving at least one injured or killed cyclist). Previous research identified intersections as more dangerous for bicyclists in comparison with other types of infrastructure [10-13]. Within the countries that report accidents location to the Community database on Accidents on the Roads in Europe (CARE) [2], 29\% of the cyclists' fatal accidents between 2005 and 2010 took place at junctions. From those, $83 \%$ took place at crossroads. Among the accidents ending in serious injury, $41 \%$ occurred at junctions, which represents a higher percentage than that of injuries occurring outside of junctions (31\%). In Italy, the $44.9 \%$ of the accidents involving a cyclist injury or death in 2011-2013 (22.294) took place at crossroads [9]. From these accidents, the majority (78.5\%) involved a passenger car and a 7.1\% involved a truck. Moreover, accidents with cars and trucks represented the $32.2 \%$ of the overall cyclist fatalities, which highlights the importance of the study and prevention on these scenarios.

The present study focuses on road conflicts between cyclists and passenger cars or goods vehicles at intersections. Among the most investigated types of maneuvers or violations associated with bicycle to motorized vehicles crashes at intersections, most of them are related to the type of accident labeled "looked-but-failed-to-see" [14, 15]. Some studies revealed that failure to see a cyclist may be due to driver inadequate scanning strategy, visual search strategies, and misplaced expectations of cyclist behavior [16-18].

Moreover, previous cycling safety research has found that left- and right-turning vehicles had an effect on bicyclist injury occurrence and were frequent in bicycle accidents taking place at intersections [16, 19-21]. These two scenarios are the so-called left turn and right turn scenarios. We will proceed to explain them with more detail below.

2.2. Left Turn Scenario. This scenario involves a vehicle turning left at an intersection and the driver failing to yield to a cyclist that approaches the intersection from the opposite direction, or getting in the trajectory of a cyclist approaching on the same direction. In both situations, the cyclist and the driver start their maneuvers on opposite sides of the road. Figure 1 displays the maneuver with a cyclist approaching on the opposite direction.

In the case of bicyclists coming in the same direction of the vehicle, blind spots or objects obstructing the view of the motorist could be a contributing factor to the failure to detect them.

2.3. Right Turn Scenario. The right turn scenario involves a vehicle failing to yield a bicyclist that rides straightforward in the same direction. It most commonly takes place when a vehicle and a bicycle arrive at the same time at an intersection with at least three branches [21]. Figure 2 shows the driver's and cyclist's maneuvers involved in the scenario.

Among truck drivers, one of the main contributing factors of this scenario could be that of failing to look/see the bicyclist going straight forward while turning [22]. Blind spots may be the cause, or the contributing factor, of this type of human error.

Cooperative systems, also exploiting infrastructure-based sensors, could be useful to improve detection of cyclists and may assist drivers in minimizing blind spots and "lookedbut-failed-to-see" errors. New technological solutions aimed at detecting bicyclists and conveying this information to the driver may play an important role in the prevention of these detection failures and, consequently, of the potential collisions.

\section{Bicyclists Detection Technologies}

The previous analysis has revealed how accidents occur due to a lack of visibility between the road users, especially at junctions and in the presence of large vehicles. This section aims at describing the main technologies capable of improving the visibility and then increasing the safety of road users. Specifically, we will distinguish between on-site and onvehicle solutions depending on the sensors' location. For each solution the advantages and disadvantages will be illustrated.

Table 1 reports some examples on the panorama of technological solutions for detection of bikes. Sensors, for example, cameras or radars, can be placed on the infrastructure (i.e., on-site) or on vehicles. Most of the detection approaches do not require technology on-bike (e.g., radar, cameras, or 
TABLE 1: Summary of available technologies.

\begin{tabular}{|c|c|c|}
\hline Technology & Pros & Cons \\
\hline Cameras & Accurate detection and users' discrimination & Difficult operation in bad weather conditions, false alarms \\
\hline Thermal cameras & Accurate detection and users' discrimination & Cost, false alarms \\
\hline Ultrasonic sensors & Reliable detection in every weather condition & Poor users' discrimination \\
\hline Inductive loops & Average users discrimination & Undersurface. Large infrastructure. Very low range \\
\hline LIDAR & Accurate detection and users' discrimination & $\begin{array}{l}\text { Very high cost, complex deployment and complex data } \\
\text { processing }\end{array}$ \\
\hline Radar (on-air) & Reliable detection. Good angular resolution & Poor users' discrimination \\
\hline Radar (under surface) & Average users' discrimination & Undersurface. Very low range \\
\hline RFID & Accurate detection and perfect users' discrimination & Tags needed. Poor angular and ranging resolution \\
\hline GPS & Localization of different users & GPS receivers needed. Medium localization accuracy, latency \\
\hline
\end{tabular}

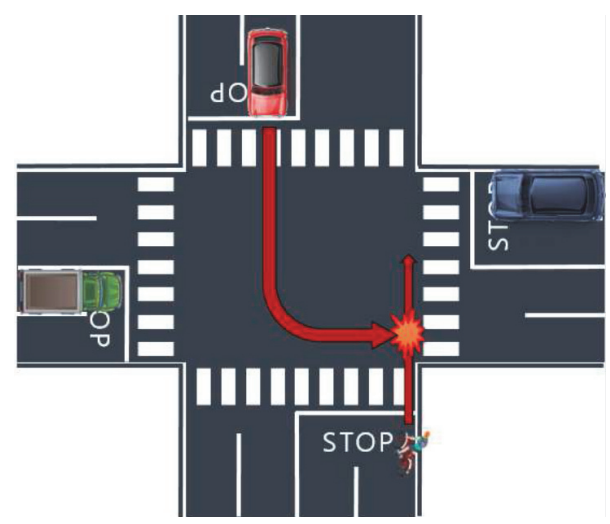

Figure 1: Left turn scenario.

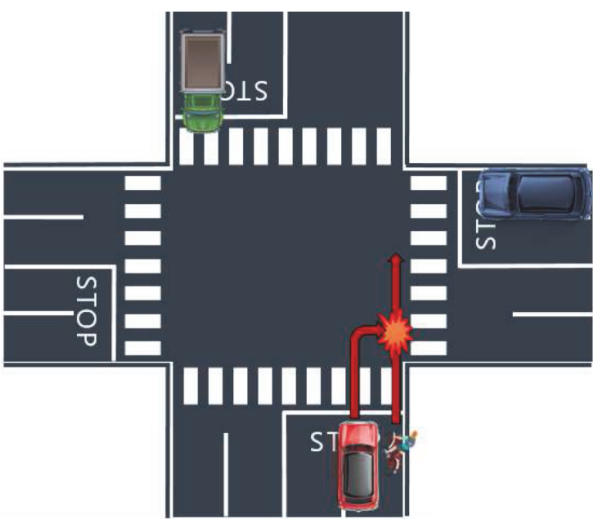

Figure 2: Right turn scenario. sonar) while others do (e.g., global positioning system (GPS) receivers or radio-frequency identification (RFID) tags).

For what concerns the characteristics of the different technologies, narrow-beam radars or sonar-based systems are efficient for detecting the presence of a user in a bicycle-path and providing an alert sign. However, they are not effective in enabling potential collision prediction or multitarget discrimination at junctions. On the other hand, cameras and especially thermocameras perform well in large road junctions and can be used for trajectory (collision) prediction. Some weather conditions make detection with normal cameras difficult (e.g., darkness or rain can decrease the capability of identifying cyclists). Thermocameras may solve, in part, this problem, but they are currently still quite expensive and might fail during the summer because the high temperature of the asphalt might hide the presence of persons. Inductive loops and undersurface radars are effective but have limited range, infrastructural problems and poorer target discrimination.

A common shortcoming of all these passive technologies is that there is always a nonnegligible probability of false alarms that may discourage users to trust the system. On the other side, the main advantage is that they do not require dedicated hardware on bike.

Other solutions, such as GPS and RFID, that require bikes equipped with dedicated technology, respectively, a GPS receiver and a RFID tag, eliminate the presence of false alarms but do not provide sufficient localization accuracy. Moreover, GPS is expensive and battery hungry and is characterized by a large response delay that prevents its use in RA.

As regards the on-vehicle technologies, they are often based on proximity sensors, camera, or radar and they usually provide warnings to other road users, such as cyclists, by means of visible alerts. Communication operations between vehicles, infrastructure, and cyclists/pedestrians are not provided by such systems as the majority of them rely on LEDs to prevent a possible collision with other road users. Moreover, the few on-vehicle solutions sometimes lack reliability in terms of misdetections and false alarms.

To conclude, the main disadvantages of the today's solutions also represent incentives for future improvements. Specifically we envisage the following desired capabilities:

(i) Submeter localization and tracking with extreme lowlatency $(<500 \mathrm{~ms})$

(ii) Possibility of detecting and discerning different road users (reducing or eliminating false alarms)

(iii) Possibility of predicting the road users' trajectories

(iv) Possibility of communicating with other road users (e.g., cyclists or pedestrians) and warning them in a specific way 
Based on the above considerations, the need of a lowcost, low-latency detection and submeter localization, and communication technology clearly emerges to support new generation RA systems capable of predicting the situation of collision and provide a suitable feedback to the vehicle and to the cyclist via ad hoc HMIs.

In the following section we propose on-site and onvehicle architectures using the UWB technology.

\section{Proposed System Architectures and Technologies}

Inspired by the shortcomings that emerged in the current available technologies, in this section we will describe a localization system capable of improving the cyclists' visibility and achieve submeter accuracy, thus guaranteeing an increased safety for the road users.

The proposed architecture is based on active, small, and low-cost tags mounted on the bikes. Contrary to their passive counterparts, active tags are equipped with a transmitting section and are able to send and/or receive interrogation signals, thus also enabling the possibility of communicating with the vehicle and/or the infrastructure and including HMI. In the future, if energy efficiency wants to be pushed at most, semipassive tags could be considered at bike side, thus minimizing the energy necessary for detection and localization and using most of the battery power just in the case of HMI activation [23, 24].

Tag detection and tracking are performed by analyzing the data exchanged by the tag and a set of reference nodes, called anchors, placed in known positions in the space, forming the so-called real-time locating systems (RTLS). In the following two architectures will be presented. The main difference of the proposed architectures lies on where anchors are deployed.

More specifically, we consider an infrastructure-based architecture with anchors placed in the correspondence of the crossing's infrastructure (e.g., at traffic lights) and a vehiclebased architecture with anchors mounted on vehicles (e.g., cars and trucks). In both solutions several anchors interact with tags by performing different kinds of measurements, as it will be clarified in Section 4.1; then a central unit gathers the information from all the anchors and fuses it to estimate the position of each tag (i.e., each bike). According to this scheme, in the first architecture the central unit is placed on the infrastructure, while in the second it is mounted on the vehicle.

In the following, we will introduce the underlying UWB technology and communication protocol, and then both architectures will be detailed.

4.1. UWB Technology and Communication Protocol. While the IEEE802.11p standard is emerging as the key technology for vehicular communications, a similar counterpart for high-accuracy positioning and tracking for intelligent transportation systems has not been presented yet. Our proposal is to consider UWB signals to achieve the target submeter positioning accuracy. In accordance with the FCC definition,

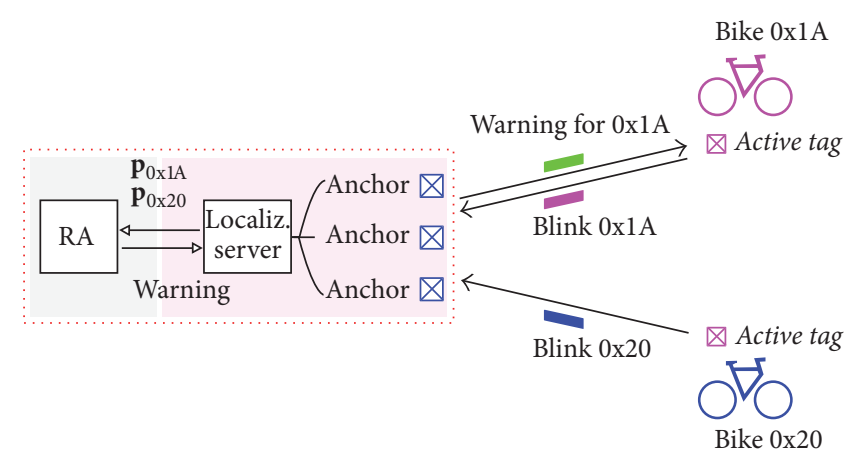

FIGURE 3: Proposed system using active UWB tags.

UWB signals are characterized by a bandwidth larger than $500 \mathrm{MHz}$. The classical and simplest method to obtain UWB signals is by means of impulse radio UWB (IR-UWB) [3]. In an IR-UWB system, a sequence of short pulses (typically with duration around $1 \mathrm{~ns}$ ) is transmitted per information bit in order to collect more energy and allow multiuser access [25]. The short duration of the pulses guarantees a fine resolution in signal time-of-arrival (TOA) measurement and multipath discrimination so that time-based localization algorithms can benefit in obtaining accurate localization estimates [6]. The UWB technology is currently utilized as baseline in highperformance short-range RTLS using active tags according to the IEEE 802.15.4a and IEEE 802.15.4f standards as well as proprietary schemes $[4,26]$.

In Figure 3, the conceptual architecture is shown. As it is possible to see, the different road users periodically transmit a blink packet following the ISO/IEC 24730-62:2013 [27] standard containing their unique 64-bit identifier (ID) to the on-site and/or on-vehicle anchors using the UWB link. In our implementation, once a tag enters the monitored area, it is detected by the anchors and the localization server assigns it a temporary 16-bit ID (tag index). Such a tag index is used to address the tag until it exits the area and it allows drastically reducing the size of the exchanged packets during the tracking process, thus reducing the risk of packets collision. The anchors receive these blink packets and, thanks to ad hoc processing schemes, determine the road users' positions and other tracking parameters (localization engine). Then, if the RA unit predicts a potential dangerous situation, a warning is sent to a specific bike in order to activate the onbike HMI.

In the following the two architectures proposed will be analyzed in order to better put in evidence their main peculiarities.

4.2. Infrastructure-Based Architecture. The infrastructurebased architecture is schematically depicted in Figure 4(a). As previously mentioned, in this solution, anchors are placed on the infrastructure, for example, at the corners of the junction in correspondence of the traffic light towers, while active tags are on bikes. Note that vehicles can be equipped with active tags as well, and, thus, they can be tracked by the system. This allows drawing a virtual map of all the road users. 


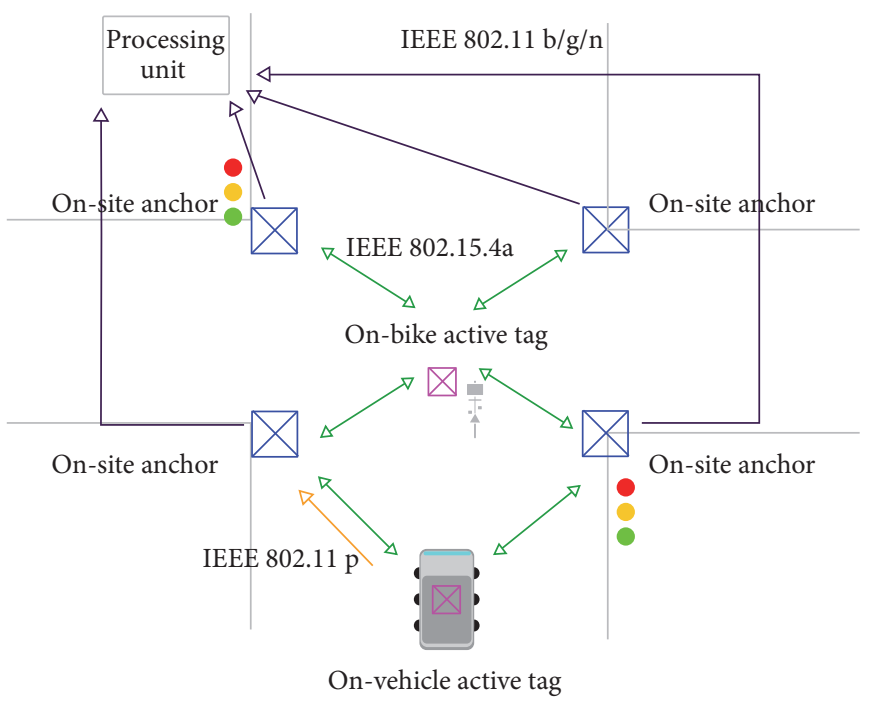

(a) Infrastructure-based architecture

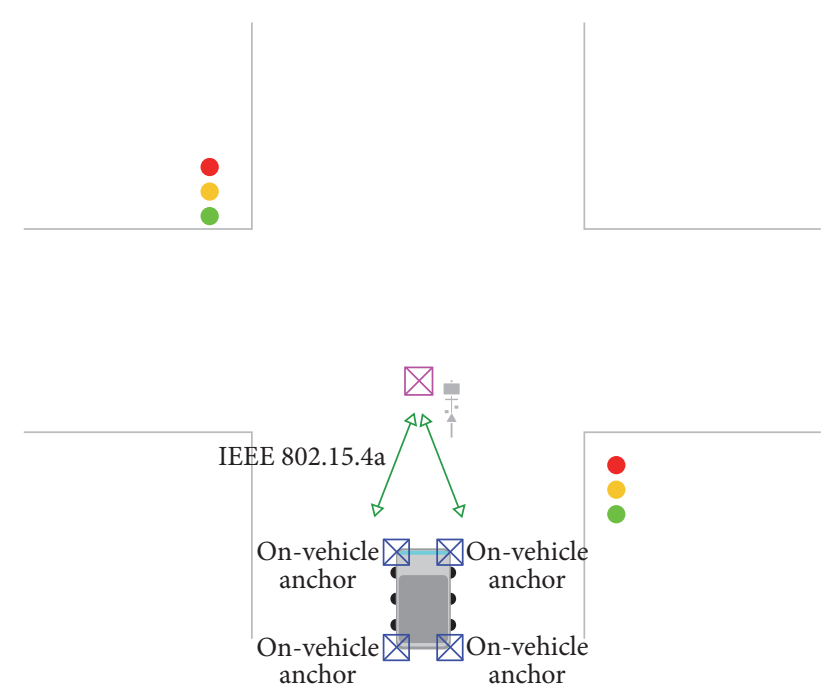

(b) Vehicle-based architecture

FIGURE 4: Localization architectures with anchors (blue) placed on the infrastructure (a) or on the vehicle (b) and tags (pink) placed on bikes and vehicles.

Given their active nature, tags periodically send interrogation signals compliant with the IEEE 802.15.4a standard to the anchors. The exchanged messages contain the ID thanks to which each road user can be discriminated. The communication between vehicles and the infrastructure can be ensured by IEEE $802.11 p$ links, whereas infrastructure-to-bike communication is enabled by the UWB IEEE 802.15.4a link.

Each anchor node receives the UWB signals from bikes and vehicles while keeping the synchronization with the other anchors. Starting from the sensed data, the processing unit performs both localization and RA process, as reported in Figure 3. The former consists in estimating the position, relative speed, and acceleration of each active tag. These data are then communicated to the RA unit whose main task is to establish the probability of collisions or more generally the presence of some risky situation. For instance, vehicles can inform the infrastructure whether they intend to start a dangerous maneuver, for example, the right and left turn, via a IEEE 802.11p link (an alternative option could be that of using the UWB technology). Based on this information and on the estimated position of all road users present in the junction, the RA unit could detect a dangerous situation and send back a warning message to the involved vehicle and to the cyclist. The on-bike device is able to activate its HMI (e.g., a visible or audible alert) to proper inform the bicyclist of the potential dangerous situation.

The main advantage of this solution is the good localization coverage that can be achieved thanks to deployment of anchors at the corners of the area to be monitored. On the other hand, this architecture is suitable in situations in which the time needed to prevent a bicycle running into a possible dangerous situation could be relaxed, as for example, in the left turn scenario, because of the potentially nonnegligible latency introduced by the vehicle-infrastructure-bike interaction.
4.3. Vehicle-Based Architecture. Figure 4(b) reports the vehicle-based architecture. Contrary to the previous described scenario, here vehicles are equipped with anchors. This means that the processing unit, consisting of the localization and RA engines represented in Figure 3, is mounted on vehicles.

The on-vehicle anchors receive the interrogation signals from the on-bike active tags in accordance with the UWB IEEE 802.15.4a standard. Thanks to these interrogations, the location engine running on-vehicle is able to determine the position and other navigation parameters (e.g., speed, acceleration) of the bikes with respect to the vehicle. Then, based on the relative coordinates of vehicle and bicycle, the RA module, still working on-vehicle, can determine whenever a dangerous situation is present or not and take proper countermeasures. As before, exploiting the same IEEE 802.15.4a links adopted for bicycle localization, the vehicle can send warnings to the on-bike tag regarding the risk and properly activate the on-bike HMI.

The main novelty of this solution is that anchors are closer to each other in comparison with the infrastructurebased approach. Therefore, the tags are always located outside the perimeter described by the anchors. As known in localization theory, this is not the optimal configuration for a localization system, since geometric dilution of precision (GDOP) issues can degrade the localization accuracy [28]. For this reason, in Section 5.3, the investigation of the impact of the geometry will be carried out, in order to understand the scale of performance degradation. On the other hand, the main advantage of such a configuration is that bicycle localization can be performed without the need of a specific infrastructure placed at the intersection and no false alarms occur. Moreover, this architecture is more promising when the decision about a possible risky situation and the relative warning message have to be rapidly conveyed to the vehicle 
driver and to the on-bike module thanks to the direct interaction between the two road users. For example, it is well suited in the right turn scenario where a stringent latency requirement has to be met.

4.4. Enhanced Services. The localization system described in the previous sections could also enable new services, as, for example, those related to green-wave scenarios. Specifically, different green-wave approaches could be implemented: one could be aimed at informing cyclists about how to adjust their speed in order to get the green light at the next junction, while another possibility is that of synchronizing traffic lights based on the number and speeds of cyclists estimated to arrive at next intersections. For this kind of application, the infrastructure-based architecture is the most promising one. Indeed, the anchor nodes placed on the crossing area can monitor the number, direction, speed, and acceleration of cyclists at the exit of the considered intersection. Based on these parameters, a dedicated processing unit can estimate the number of bikes and the time needed to arrive at the next junction and thus the traffic lights can be programmed accordingly to allow a safer transit of cyclists. The mechanism through which the cyclists are localized and tracked is the same as described in Section 4.2.

\section{The UWB Tracking Subsystem}

In this section, the RTLS proposed and implemented for this application is described. As already introduced, the localization process is based on the capability of extrapolating positioning information starting from the UWB signals exchanged between nodes. Specifically, anchors receive the interrogation signals compliant with the IEEE 802.15.4a standard sent by active tags powered by an on-board battery and including a radio transceiver with transmitting/receiving capability.

5.1. On-Bike Module. The on-bike module implemented, shown in Figure 5, is mainly composed of an IEEE 802.15.4a transceiver connected to a UWB antenna. The transceiver communicates via Serial Peripheral Interface (SPI) with a MCU. The microcontroller unit (MCU) determines the sensor module behavior and implements all the functionalities needed for enabling localization and communication with vehicle and/or infrastructure. Output ports of the microcontroller unit (MCU) are then adopted for activating the onbike HMI. As reported in the figure, the on-bike module is also equipped with additional $3 \mathrm{D}$ sensors (i.e., 3-axis accelerometer, 3-axis gyroscope, 3-axis magnetometer, and pressure sensor) whose output can be processed by the RA unit. All the sensors are periodically interrogated by the microcontroller unit (MCU). After the interrogation cycle, the sensor outputs are sent via the UWB link as payload of the blink packets to the anchors and thus made available for the location process running on vehicle or on infrastructure. The outputs of the localization estimation process consist of the bicycle ID, position coordinates, velocity, acceleration, absolute orientation, and the expected positioning accuracy.

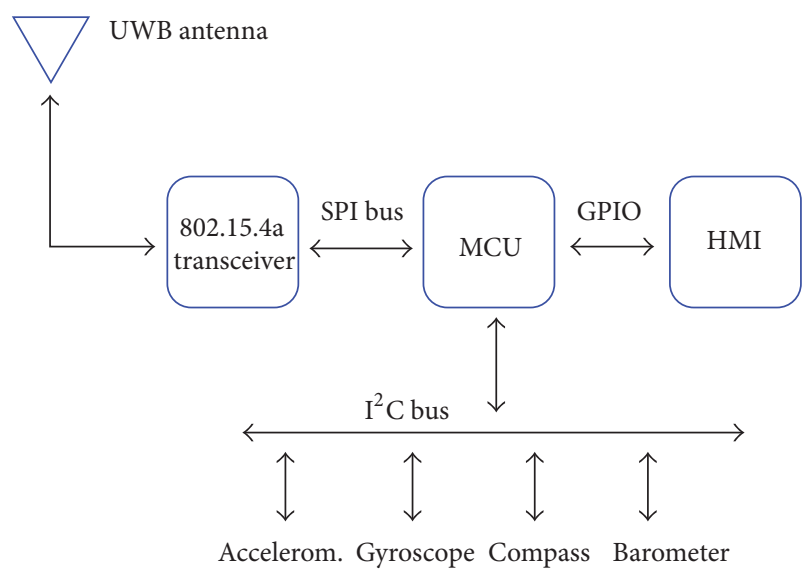

FIGURE 5: On-bike tag module.

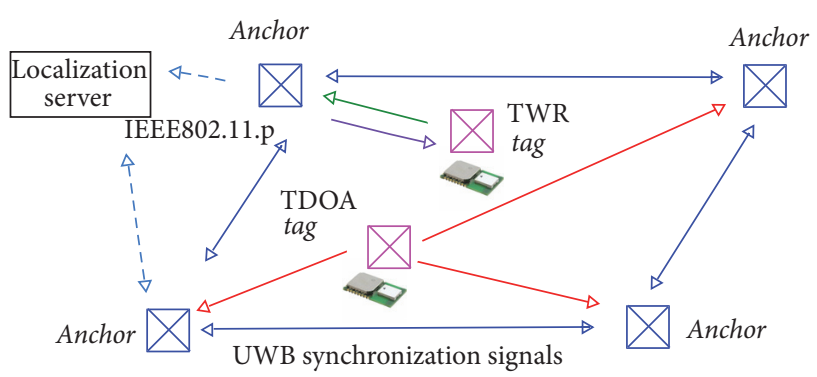

FIGURE 6: Localization scheme using TDOA and TWR tags.

The on-bike module also contains an HMI to proper inform the bicyclist of a potential danger predicted by the RA unit (either considering bicycle-vehicle interaction or bicycle-infrastructure interaction or simple detection by onvehicle or infrastructure anchors). The bicycle HMI should be designed such that it requires minimal attention and does not distract the cyclist. Examples of HMIs are a flashing light display, an auditory alert, or a combination of both. The implementation of these should take into account the lighting conditions and background noise when cycling next to a truck. Other possibilities are, for example, HMIs based on helmet or handlebar vibrations.

5.2. Localization and Tracking Methods. The considered UWB RTLS is capable of providing submeter localization accuracy without resorting to costly and less accurate technologies such as GPS. The system deployment mainly consists on the installation of a sufficient number of anchors placed in known positions. The number of anchors depends on the area to be covered: the larger the space where tags can be placed is, the greater the number of anchor nodes should be to guarantee an acceptable level of tracking accuracy.

The main approaches to infer tag's position resort to TOA measurements as done in two-way ranging (TWR) and time difference-of-arrival (TDOA), as shown in Figure 6 and more schematically in Figure 7 [28]. TWR is based on packet exchanges between a couple of nodes (e.g., with reference to Figure $7(\mathrm{a})$, the tag, node $\mathrm{B}$, and one of the anchors, node 


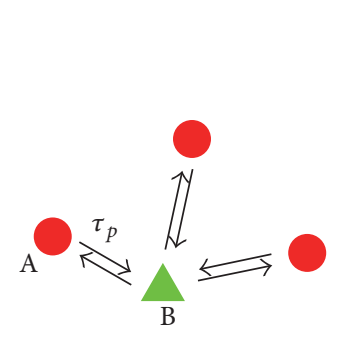

(a) TWR

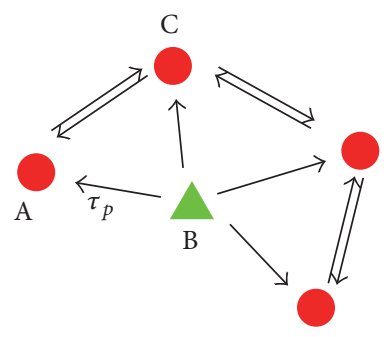

(b) TDOA
FIGURE 7: TWR and TDOA localization techniques.

A). The round-trip time of this ping-pong, $\tau_{\mathrm{RT}}=2 \tau_{p}+\tau_{d}$, is measured with $\tau_{p}$ being the time of flight and $\tau_{d}$ the response delay, so that distance is estimated (ranging) [29]. This process must be repeated for any anchor and tag couple. Considering at least three anchors performing the TOA measure, it is possible to estimate the position of the device resolving a triangulation problem.

Differently, the TDOA principle lies on the idea of estimating the position of the transmitting tag by using the difference in time at which the signal arrives at multiple anchors. Therefore, the tag is expected to be placed on a hyperbole where the anchors are in the foci of the curve and where the distance between them is a priori known [30]. Note that, in this case, anchors exchange the collected time-based measurements, as reported in Figure 7(b). Having at least three anchors (i.e., two TDOA measurements) it is possible to estimate the position of the tag. This requires a very accurate synchronization among nodes that has to be guaranteed by distributing a common clock via cable or wirelessly.

If from one side, TWR provides a higher positioning accuracy thanks to the possibility of better counteract oscillators clock drifts, from the other side TDOA permits a drastic reduction in packet exchanges between tags and anchors, thus allowing a significant improvement in terms of refresh rate and multiple tags management. In fact, in TDOA each tag sends a very short blink packet which is received by the network of anchors. Due to the short duration of the blink packet emitted, a large number of tags can be managed at the same time, with both random channel access (e.g., aloha-based solutions) or time-scheduled transmissions (e.g., time division multiple access (TDMA)). In this manner, differently from the TWR scheme, a single uplink taganchor is sufficient for positioning (the downlink channel is then used only for infrastructure-to-bike or vehicle-to-bike communication). This solution allows building an extremely simple architecture for the tag, since all the heavy processing for localization is demanded to the central unit placed on infrastructure or vehicle. Moreover, the short blink packets contribute in maintaining the power consumption at tag side low, thus enabling battery-powered, low-cost solutions.

We implemented both the TDOA (with wireless synchronization) and TWR to make some comparisons in terms of performance, as it will be presented in the numerical results. At central unit side, based on the collected measurements, the tag position calculation is performed through state-of-the-art

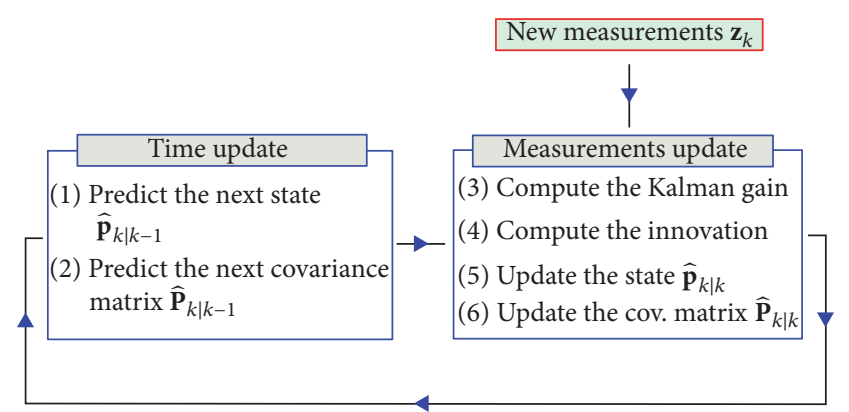

Figure 8: Bayesian filtering iterations.

Bayesian filters. Specifically, we implemented a Bayesian filter using the particle filter approach employing measurements fusion mechanisms and clock drift compensation techniques to enable TDOA. The overall latency is less than $500 \mathrm{~ms}$.

Figure 8 shows the classical two steps constituting the Bayesian filtering iterations. As it can be seen, the main objective is to infer an estimate of the state (e.g., tag's position coordinates, absolute orientation, and velocity) and its covariance matrix (i.e., a measure of the estimation uncertainty). More specifically, the first step consists in performing a prediction based on the history of the collected measurements (time update). Once new measurements become available (measurement update), an estimation correction phase can be undertaken based on the computed Kalman gain which is an indicator of the measurements and prior predictions reliability. In our approach, the observation is modeled as a unimodal Gaussian distribution centered in the difference between the estimated TDOA and the true TDOA and with a TOA error standard deviation of $0.2 \mathrm{~m}$ characterized from measurements. Regarding the mobility model, we assumed two different solutions depending on the availability of the speed information. In the speed-unawareness case, the density function describing the mobility model corresponds to a Gaussian distribution centered in the previous estimated position, whereas in the second case a speed learning approach is adopted where the speed is evaluated from earlier estimated positions with a sliding window. For further details, the reader is invited to refer to [31]. Note that, if tags broadcast their sensor data, inertial measurements fusion techniques can be adopted for improved localization accuracy [31].

\subsection{Impact of Architecture on the Localization Accuracy:} Performance Limits. As anticipated in Section 4, a discussion about the anchor nodes placement is necessary. In fact, it is well known from the localization literature how the geometrical configuration of anchors impacts the localization accuracy. Such effect, known as GDOP, arises when combining different measurements and can worsen or improve the performance in given position of the space where the tag could be present.

In order to access the potential localization accuracy, in particular in the vehicle-based infrastructure which is known unfavorable from the GDOP point of view, a theoretical analysis based on the position error bound (PEB) [32] is 


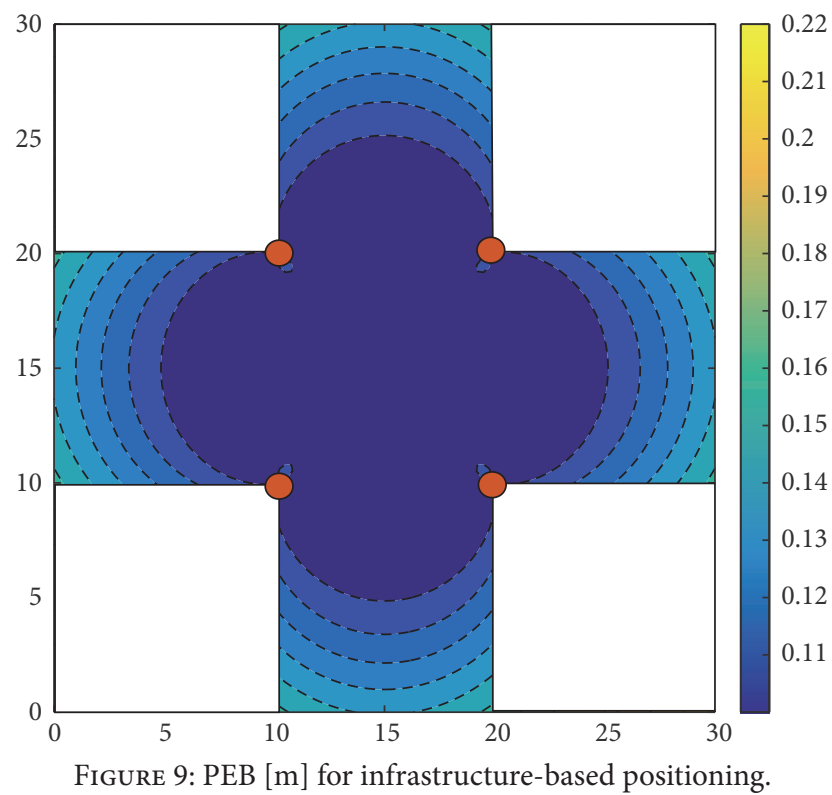

here carried out. PEB is a tool for determining what is the theoretical best positioning accuracy that an algorithm can provide starting from a certain setting in terms of measurements and geometrical configuration of anchors and tag. Specifically, it is defined as

$$
\operatorname{PEB}(\mathbf{p})=\sqrt{\operatorname{tr}\left(\mathbf{J}^{-1}(\mathbf{p})\right)},
$$

where $\mathbf{p}$ is the tag position, $\operatorname{tr}(\cdot)$ is the trace operator, and $\mathbf{J}(\mathbf{p})$ is the positioning Fisher information matrix (FIM). Following [32], it is possible to obtain

$$
\mathbf{J}(\mathbf{p})=\Gamma \sum_{i=1}^{N} \mathbf{G}\left(\boldsymbol{\Theta}_{i}\right)
$$

where $N$ is the number of independent measurements (i.e., the number of anchor nodes), $\Gamma=f\left(\sigma^{2}\right)$ with $\sigma^{2}$ being the variance of the ranging observation noise, then carrying information on the measurements quality, and $\mathbf{G}(\cdot)$ is the geometric matrix related to the GDOP with $\boldsymbol{\Theta}_{i}$ representing the $i$ th anchor direction, then carrying information about the anchors' placement. Note that, given the FIM in (2), it is possible to optimize the anchors deployment in order to maximize the informative content of the measurements and thus the localization accuracy. Therefore, an accurate analysis of the best anchors' deployment is strictly related to the optimization of the GDOP function. Moreover, as previously mentioned, from (1) to (2), it is possible to understand how the number of anchors (i.e., $N$ ) impacts the localization performance.

In Figure 9 the PEB for the infrastructure-based architecture is shown. Specifically, a standard deviation of $20 \mathrm{~cm}$ for ranging is considered, and the presence of 4 anchors placed in the correspondence of the traffic lights in a $30 \times 30 \mathrm{~m}^{2}$ junction. Anchors are depicted with brown circles and are placed in coordinates $(20,10),(20,20),(10,20)$, and $(10,10)$. From the figure, it is possible to see how the localization error is equalized in the entire scenario, always between

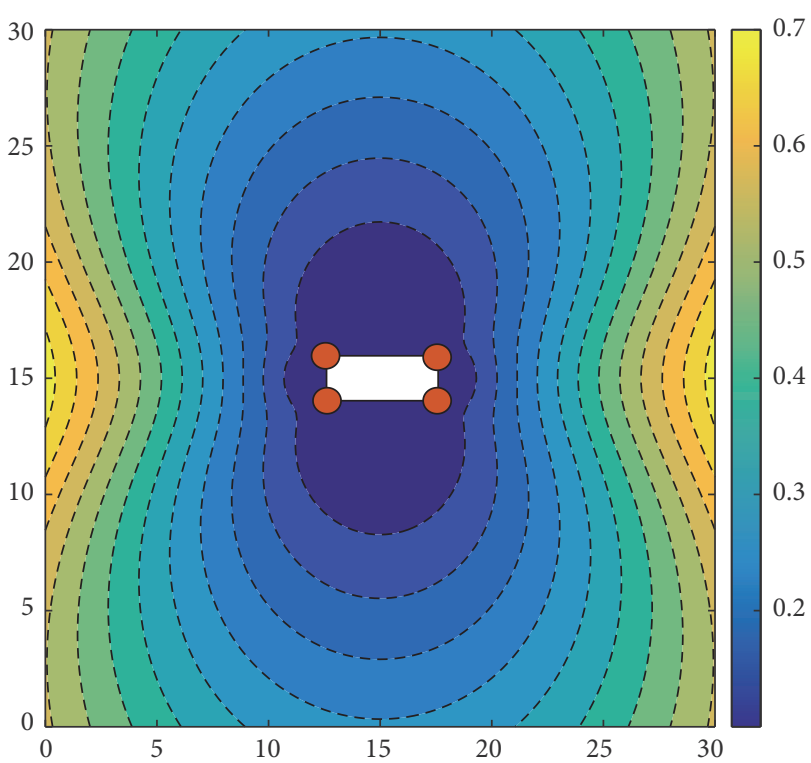

FIGURE 10: PEB [m] for vehicle-based positioning (small truck).

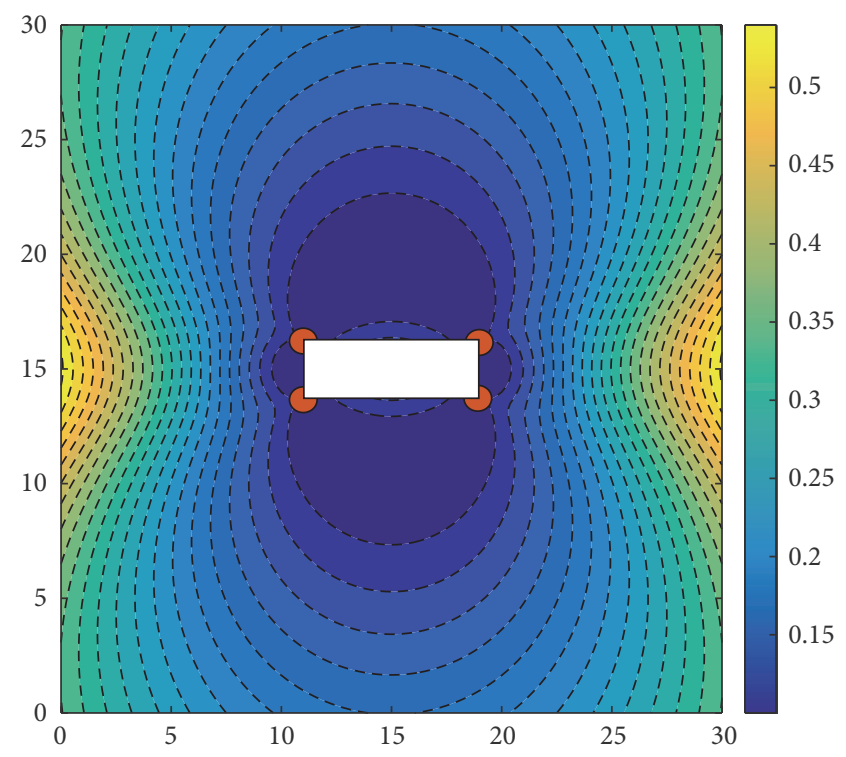

FIgURE 11: PEB [m] for vehicle-based positioning (large truck).

10 and $20 \mathrm{~cm}$. On the other hand, Figures 10 and 11 show the PEB for the vehicle-based architecture. Again, a ranging standard deviation of $20 \mathrm{~cm}$ is considered with the presence of 4 anchors placed at the corners of (i) a vehicle (small truck) of size $2 \times 5 \mathrm{~m}^{2}$ and (ii) vehicle (medium-sized truck) of size $2.5 \times 8 \mathrm{~m}^{2}$. In both cases the truck is assumed in the middle of the scenario. From the figures, it is possible to notice the GDOP effects, which are more pronounced in the direction of the vehicle. In fact, the localization error increases moving away from the vehicle, and such performance degradation is less severe in the orthogonal direction. This is mainly due to the geometrical placement of the anchors along the long vehicle. When adopting vehicle-based architectures, it is then fundamental to consider location estimates only in a certain area of some meters around the vehicle, where the location process is effective. 

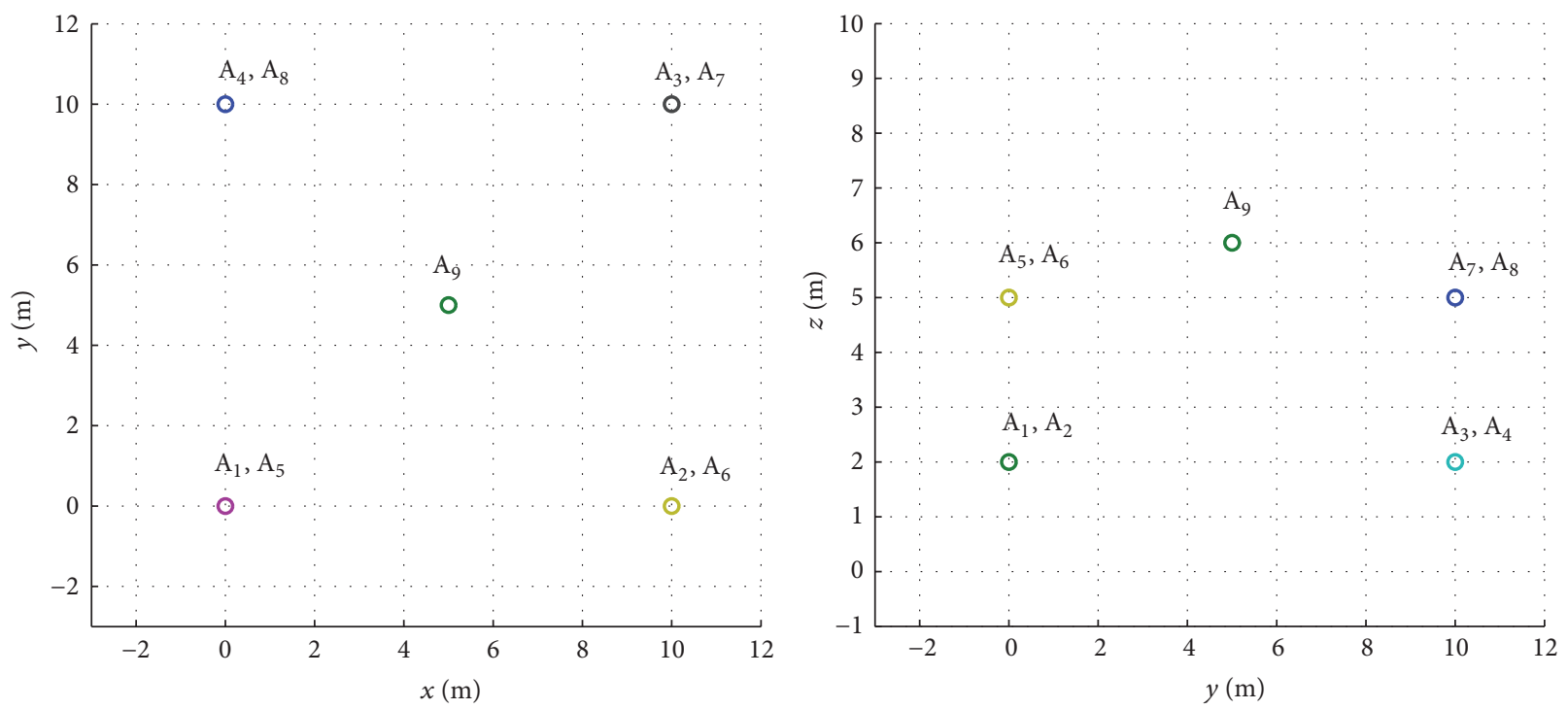

FIGURE 12: Anchors deployment: $x-y$ plane on the left and $y-z$ plane on the right.

It is important to notice that the final localization accuracy is also impacted by propagation conditions, in particular by the presence of non-line-of-sight (NLOS) channels between tags and anchors [33-35]. In fact, due to the time-based localization technique proposed, an obstacle can interact in several manners with the radio signal used, for example, for ranging. One typical effect is the obstruction of the direct path so that TOA estimation is wrongly performed on a reflected path, then relating to a larger distance with respect to the optical ray. In other cases, also if the direct path is not completely blocked, the propagation inside a different medium slows down the electromagnetic wave. In both cases, NLOS channel conditions cause an overestimation of the distance and consequently lead to a decreased positioning accuracy [28].

In our scenario, such conditions could occur for several reasons. In an infrastructure-based solution, NLOS condition could be caused by the presence in the crossing area of large trucks blocking the tag-to-anchor signal. Due to the particular characteristic of such a scenario, where the obstruction of other vehicles change rapidly with time (differently from traditional NLOS conditions caused, for example, by walls or furniture), the investigation of the impact of such effects requires ad hoc measurements in real dynamic conditions. Such an investigation is part of our future experimental activity even though redundancy appears the most viable solution in rapidly changing scenarios. Differently, in the vehicle-based solution, the NLOS channel could arise due to the intrinsic shape of the vehicle, which obstructs some of the anchors placed on it. When NLOS channel conditions are present, the localization accuracy decreases and ambiguities in the position can arise due to an insufficient number of measurements. However, thanks to the considered tracking scheme, where a location engine continuously tracks a given tag starting from new measurements and the prior location estimation, ambiguities can be strongly mitigated. Moreover, NLOS conditions can be reduced by deploying a larger number of anchors nodes and by fusing inertial data. Notice that, thanks to the considered TDOA scheme, this translates only on an increased complexity at the processing unit side, with no modifications required at tag side and no additional complexity.

\section{Experimental Results}

In this section preliminary experimental results using the implemented localization system are described in order to assess the localization accuracy in static and dynamic scenarios. The purpose is also to analyze the performance degradation when considering TDOA with wireless synchronization instead of TWR. The measurement campaigns have taken place in the CASY (Center of Complex Automated Systems) indoor flight arena at the University of Bologna premises and in a controlled junction in Cesena, Italy.

6.1. Indoor Measurements and Results. The measurements have taken place in an area of $11 \times 11 \times 6 \mathrm{~m}^{3}$ where tags can move on the $x-y$ plane with a $z$ coordinate that ranges from 1 to $2 \mathrm{~m}$. A set of 22 infrared cameras (VICON Bonita 10), installed in the monitored area, have been adopted to infer the exact tags' positions as they can achieve a millimeter localization accuracy. 9 anchor nodes are considered, according to the deployment represented in Figure 12. Specifically, we have considered the following deployments:

(i) All anchors: when all the reference nodes $\left(\mathrm{A}_{i} i=\right.$ $1,2, \ldots, 9)$ are active

(ii) Lower circle: only $\mathrm{A}_{i} i=1,2, \ldots, 4$

(iii) Upper circle: only $\mathrm{A}_{i} i=5,6, \ldots, 8$

(iv) Mixed: mixed combination between lower and upper circle: for example, $A_{1}, A_{3}, A_{6}, A_{8}$

As mentioned before, the number of active anchor nodes is one of the parameters that determine the localization 

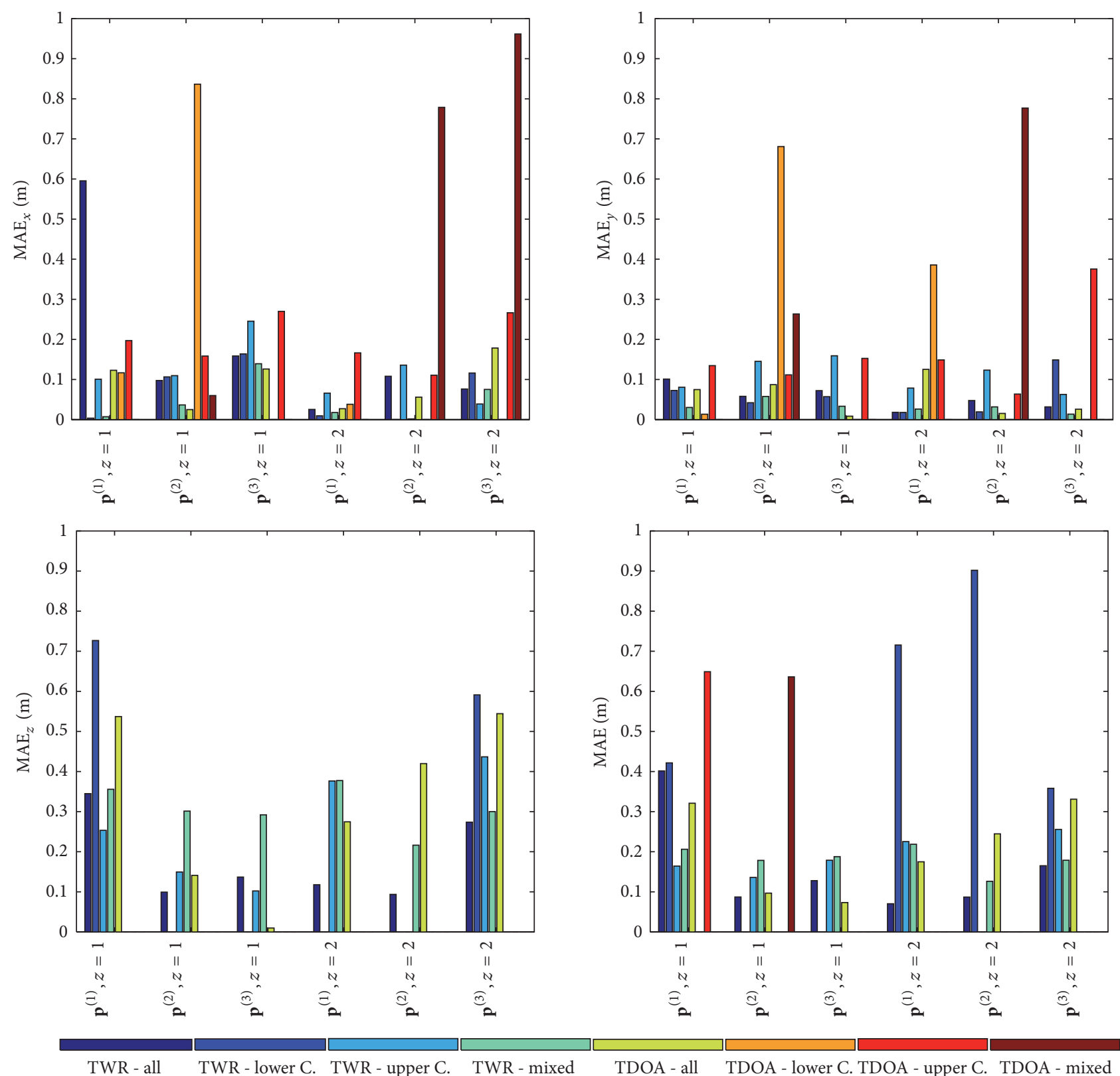

FIGURE 13: Localization error considering static tags.

accuracy of the system. For this reason, a preliminary study concerned the localization performance as a function of the number of anchors in static conditions, that is, with the tag placed in a set of fixed positions. For each test position in space, 500 estimates have been collected using a personal computer running the localization algorithm based on Bayesian filtering.

6.1.1. Results. The performance metric adopted for assessing the localization performance is the mean absolute error (MAE) defined as

$$
\mathrm{MAE}=\frac{1}{N} \sum_{i=1}^{N}\left\|\mathbf{p}-\widehat{\mathbf{p}}_{i}\right\|,
$$

where $\mathbf{p}$ represents the true tag position as derived by the VICON system while $\widehat{\mathbf{p}}_{i}$ is the estimated position. $N$ represents the number of estimates for each set of measurements.

In static conditions, the tag is placed in different fixed unknown positions inside the area. In the following, we will indicate with $\mathbf{p}^{(j)}$ with $j=1,2,3$ the three testing positions in the $x-y$ plane while as regards to the $z$ coordinate, two different heights, that is, $z=1 \mathrm{~m}$ and $z=2 \mathrm{~m}$, have been taken into account. For each tag's position, we aim at analyzing the localization performance as a function of the number of anchors and of the chosen localization technique.

Figure 13 reports the MAE for each tag's position and coordinate as function of different anchors' deployments and 
TABLE 2: Localization error (RMS) averaged over different tag positions.

\begin{tabular}{lccccc}
\hline Loc. technique & Anchors config. & $x$ error $[\mathrm{m}]$ & $y$ error $[\mathrm{m}]$ & $z$ error $[\mathrm{m}]$ & 0.20 \\
TWR & All anchors & 0.26 & 0.06 & 1.15 & 0.15 \\
TWR & Lower circle & 0.44 & 0.07 & 2.55 & 0.83 \\
TWR & Upper circle & 0.13 & 0.11 & 0.31 & 0.74 \\
TWR & Mixed & 0.06 & 0.03 & 0.37 & 0.18 \\
TDOA & All anchors & 0.10 & 0.07 & 7.49 & 0.20 \\
TDOA & Lower circle & 1.41 & 1.22 & 6.23 & 3.34 \\
TDOA & Upper circle & 0.20 & 0.19 & 5.44 & 3.13 \\
TDOA & Mixed & 1.97 & 2.23 & & \\
\hline
\end{tabular}

localization approaches. Only anchors geometric configurations resulting in a submeter localization error are reported. As one can observe, the TWR technique is more accurate in estimating the $x$ and $y$ coordinates and then TDOA, and this is particularly verified for anchors at the same height. Such degradation of the localization performance in TDOA is also due to anchors synchronization mismatches but it can be counteracted using an increased number of anchors. When considering a proper anchors deployment TDOA results are satisfactory for the application under consideration.

Table 2 reports the mean square error averaged over the tag positions and shows how the UWB localization system is able to infer the tag positions with centimeter localization accuracy also in indoor environments, especially when operating in TWR fashion and with a high number of anchors. We expect that in outdoor conditions the performance could improve because of the absence of strong multipath caused by signal reflections on the walls as indoor.

In dynamic conditions, the tag was free to move in the flight arena. During this test the tag did not follow only a longitudinal path but also performed rotational movements and modified its height. The average speed of the tag is $4.5 \mathrm{~km} / \mathrm{h}$ and the distance covered is approximately $100 \mathrm{~m}$.

Figure 14 reports the true and estimated tag trajectories. It is possible to observe that the estimated trajectory, marked with red circles, diverges from the true one, indicated with a dashed blue curve, especially in correspondence of rapid changes of direction. Nevertheless, this inconvenience could be solved by refining the mobility model in the Bayesian tracking algorithm which is one of the future tasks.

6.2. Outdoor Measurements and Results. The measurements have taken place in an area of approximately $40 \times 70 \mathrm{~m}^{2} .5$ anchors nodes have been located on a junction as illustrated in Figure 15, left by blue-squared markers, at a height of $\approx 2.90 \mathrm{~m}$. A bike has been equipped with an active tag. The aim of the outdoor measurements campaign has been to qualitatively assess the performance of the UWB tracking system and to analyze the impact of the HMI on the cyclist behavior considering the infrastructure-based scenario. In fact, either the left turn or right turn scenario has been emulated twice: the first time the HMI, consisting of a buzzer and a led, was not activated while the second time, it was remotely enabled in order to warn the cyclists of the

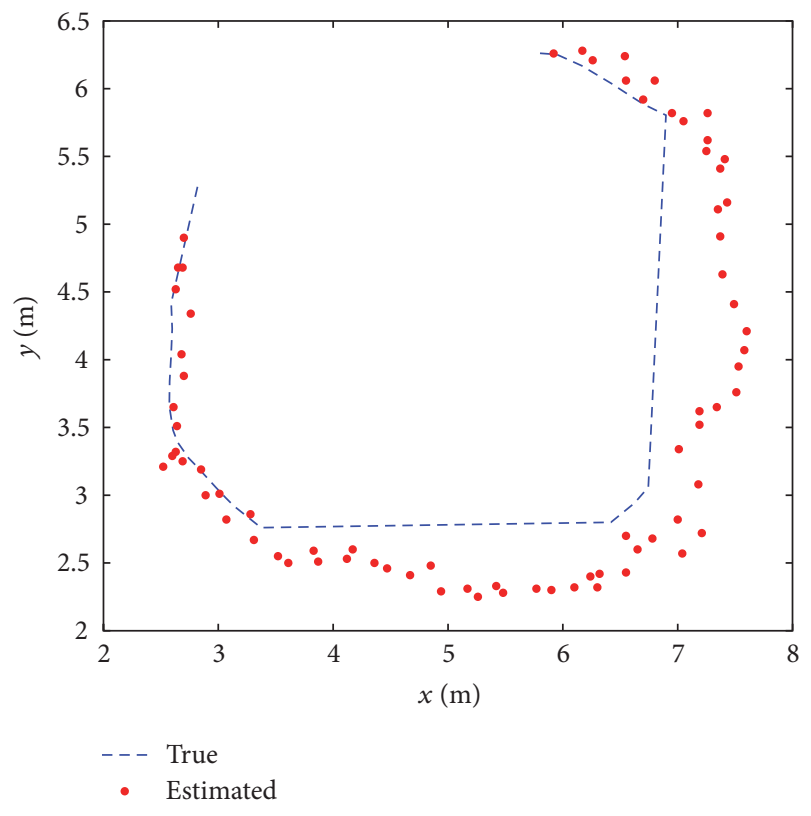

FIGURE 14: Localization error example for a dynamic tag.

approaching vehicle. In both situations, the position and the velocity of the cyclists have been recorded over time. About 20 candidates participated in the experiments and undergo a survey to test their opinions and acceptance on the adopted technology.

Figure 15 reports an example of results for one candidate. On the top-left, the cyan-colored markers indicate the cyclist's trajectory while on the bottom-left, the latter are interleaved with red markers indicating the spatial points relative to the activation of the user's HMI. As expected, the HMI is enabled in the correspondence of the junction, that is, where a dangerous situation for the cyclists could happen. On the right, the recorded speeds of the two situations are displayed. As it is evident, the cyclists behavior (in this case, its speed) sharply changed after the activation of the HMI enhancing its safety in approaching the intersection. The next activity foresees the assessment of the performance of the vehiclebased architecture and the integration of a RA unit which can infer the risk based on previous positions estimates in order to automatically activate the HMI. 

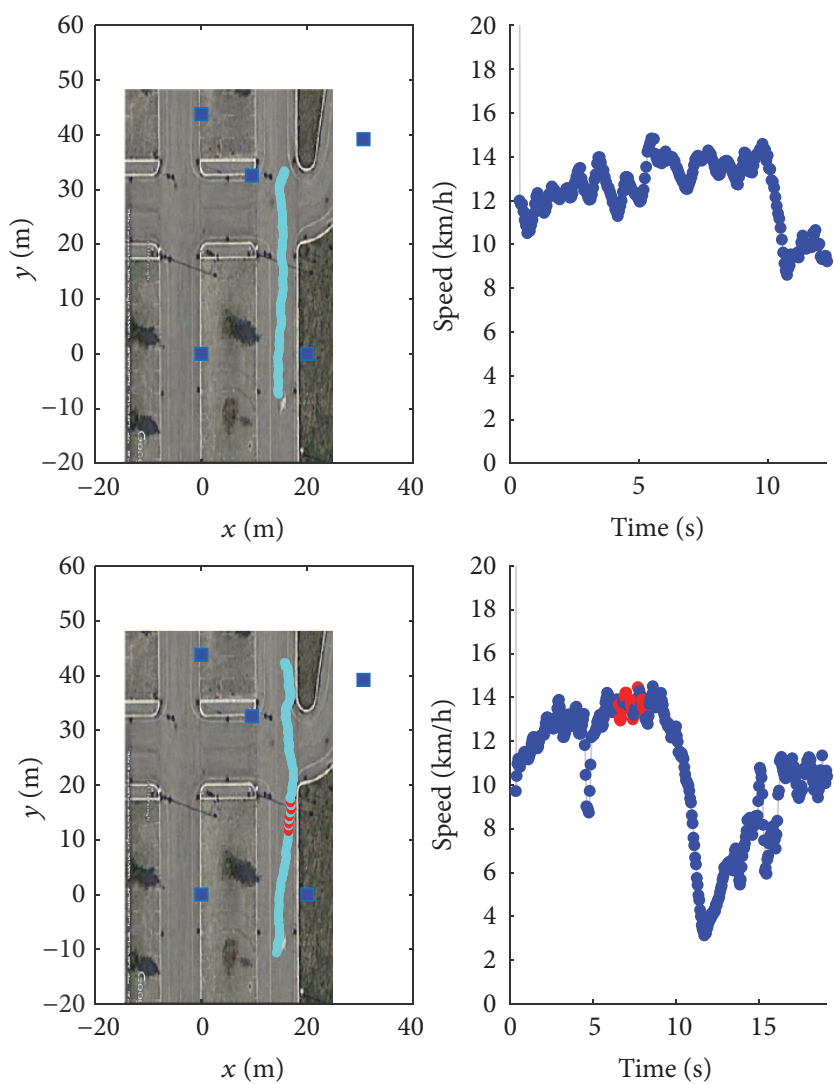

FIGURE 15: Example of outdoor measurements result.

\section{Conclusions and Future Perspectives}

Starting from a deep analysis of road users' behavior and of typical dangerous situations, in this paper two architectures performing RA and providing a feedback to the road users and, in particular, to cyclists, on potentially dangerous situations have been presented. To enable such architectures, we proposed a localization system based on the UWB technology capable of guaranteeing high-accuracy localization and tracking, in which bikes are equipped with low-cost and low-complexity active tags and with HMI. Reference nodes can be placed both at the junctions (infrastructurebased architecture) or on vehicle (vehicle-based architecture). Preliminary experimental activities have been conducted to assess the feasibility of the proposed solutions in static and dynamic conditions. Results show the possibility of achieving submeter localization accuracy and good tracking capabilities, even in harsh propagation environments.

In the future, the behavioral investigation will take a central role in evaluating different HMI solutions, to alert cyclists of dangerous situations in order to enhance their comfort and safety, without being source of distractions or annoying the final users, thus establishing the technology acceptance.

\section{Competing Interests}

The authors declare that there is no conflict of interests regarding the publication of this paper.

\section{Acknowledgments}

The authors would like to thank Federica Zonzini, Nicola Mimmo, Jurgis Aleksandravičius, Beatrice Borghetti, and Ahmed Elzanaty for the cooperation in the experimental characterization of the performance and Lorenzo Marconi for the concession of the DEI-CASY flight arena and the VICON facility. This work has received funding from the EU Horizon 2020 Research and Innovation Programme under the project XCycle (Grant no. 635975).

\section{References}

[1] ERSO, "Traffic safety basic facts 2015: cyclists," Tech. Rep., European Road Safety Observatory, 2015.

[2] European Commission, "CARE database," http://ec.europa.eu/ transport/road_safety/specialist/index_en.htm.

[3] M. Z. Win, D. Dardari, A. F. Molisch, W. Wiesbeck, and J. Zhang, "History and applications of UWB," Proceedings of the IEEE, vol. 97, no. 2, pp. 198-204, 2009.

[4] "IEEE standard for information technology-telecommunications and information exchange between systems - local and metropolitan area networks - specific requirement part 15.4: Wireless medium access control (MAC) and physical layer (PHY) specifications for low-rate wireless personal area networks (WPANs)," IEEE Std 802.15.4a-2007 (Amendment to IEEE Std 802.15.4-2006), pp. 1-203, 2007.

[5] H. Soganci, S. Gezici, and H. V. Poor, "Accurate positioning in ultra-wideband systems," IEEE Wireless Communications, vol. 18, no. 2, pp. 19-27, 2011.

[6] D. Dardari, A. Conti, U. Ferner, A. Giorgetti, and M. Z. Win, "Ranging with ultrawide bandwidth signals in multipath environments," Proceedings of the IEEE, vol. 97, no. 2, pp. 404425, 2009.

[7] Y. Shen and M. Z. Win, "Fundamental limits of wideband localization-Part I: a general framework," IEEE Transactions on Information Theory, vol. 56, no. 10, pp. 4956-4980, 2010.

[8] E. T. S. Council, "Making walking and cycling on europes roads safer," Tech. Rep. (PIN Flash 29), 2015.

[9] ISTAT, "Survey on road accidents resulting in death or injury," http://www.istat.it/en/archive/87804.

[10] S. Kaplan and C. Giacomo Prato, "A spatial analysis of land use and network effects on frequency and severity of cyclistmotorist crashes in the copenhagen region," Traffic Injury Prevention, vol. 16, no. 7, pp. 724-731, 2015.

[11] N. T. R. Romanow, A. B. Couperthwaite, G. R. McCormack, A. Nettel-Aguirre, B. H. Rowe, and B. E. Hagel, "Environmental determinants of bicycling injuries in Alberta, Canada," Journal of Environmental and Public Health, vol. 2012, Article ID 487681, 12 pages, 2012.

[12] M. Stone and J. Broughton, "Getting off your bike: cycling accidents in Great Britain in 1990-1999," Accident Analysis \& Prevention, vol. 35, no. 4, pp. 549-556, 2003.

[13] F. Wei and G. Lovegrove, "An empirical tool to evaluate the safety of cyclists: community based, macro-level collision prediction models using negative binomial regression," Accident Analysis and Prevention, vol. 61, pp. 129-137, 2013.

[14] M.-B. Herslund and N. O. Jørgensen, "Looked-but-failed-tosee-errors in traffic," Accident Analysis and Prevention, vol. 35, no. 6, pp. 885-891, 2003. 
[15] A. Koustanaï, E. Boloix, P. Van Elslande, and C. Bastien, "Statistical analysis of 'looked-but-failed-to-see' accidents: highlighting the involvement of two distinct mechanisms," Accident Analysis and Prevention, vol. 40, no. 2, pp. 461-469, 2008.

[16] M. Räsänen and H. Summala, "Attention and expectation problems in bicycle-car collisions: an in-depth study," Accident Analysis and Prevention, vol. 30, no. 5, pp. 657-666, 1998.

[17] M. Räsänen, H. Summala, and E. Pasanen, "The safety effect of sight obstacles and road-markings at bicycle crossings," Traffic Engineering \& Control, vol. 39, no. 2, pp. 98-102, 1998.

[18] H. Summala, E. Pasanen, M. Räsänen, and J. Sievänen, "Bicycle accidents and drivers' visual search at left and right turns," Accident Analysis \& Prevention, vol. 28, no. 2, pp. 147-153, 1996.

[19] R. Aldred and S. Crosweller, "Investigating the rates and impacts of near misses and related incidents among UK cyclists," Journal of Transport and Health, vol. 2, no. 3, pp. 379393, 2015.

[20] L. F. Miranda-Moreno, J. Strauss, and P. Morency, “Disaggregate exposure measures and injury frequency models of cyclist safety at signalized intersections," Transportation Research Record, no. 2236, pp. 74-82, 2011.

[21] W. Niewoehner and F. A. Berg, "Endangerment of pedestrians and bicyclists at intersections by right turning trucks," Statistics, National Highway Traffic Safety Administratio, 2005.

[22] HVU, "accidents between trucks turning right and bicycles going straight ahead," Tech. Rep., Danish Road Traffic Accident Investigation Board, Copenhagen, Denmark, 2006.

[23] N. Decarli, F. Guidi, and D. Dardari, "Passive UWB RFID for tag localization: architectures and design," IEEE Sensors Journal, vol. 16, no. 5, pp. 1385-1397, 2016.

[24] D. Dardari, N. Decarli, A. Guerra, and F. Guidi, "The future of ultra-wideband localization in RFID," in Proceedings of the IEEE International Conference on RFID (RFID '16), pp. 1-7, Orlando, Fla, USA, May 2016.

[25] M. Z. Win and R. A. Scholtz, "Impulse radio: how it works," IEEE Communications Letters, vol. 2, no. 2, pp. 36-38, 1998.

[26] Z. A. Lu, S. Gezici, and G. Ismail, Ultra-Wideband Positioning Systems: Theoretical Limits, Ranging Algorithms, and Protocols, Cambridge University Press, 2008.

[27] “ISO/IEC 24730-62:2013 standard," 2013.

[28] D. Dardari, E. Falletti, and M. Luise, Satellite and Terrestrial Radio Positioning Techniques: A Signal Processing Perspective, Academic Press, Cambridge, Mass, USA, 2011.

[29] Z. Sahinoglu, S. Gezici, and I. Guvenc, Ultra-Wideband Positioning Systems, Cambridge University Press, New York, NY, USA, 2008.

[30] B. T. Fang, "Simple solutions for hyperbolic and related position fixes," IEEE Transactions on Aerospace and Electronic Systems, vol. 26, no. 5, pp. 748-753, 1990.

[31] D. Dardari, P. Closas, and P. M. Djuric, "Indoor tracking: theory, methods, and technologies," IEEE Transactions on Vehicular Technology, vol. 64, no. 4, pp. 1263-1278, 2015.

[32] D. B. Jourdan, D. Dardari, and M. Z. Win, "Position error bound for UWB localization in dense cluttered environments," IEEE Transactions on Aerospace and Electronic Systems, vol. 44, no. 2, pp. 613-628, 2008.

[33] L. Mucchi, A. Sorrentino, A. Carpini, M. Migliaccio, and G. Ferrara, "Physically-based indicator for identifying ultrawideband indoor channel condition," IET Microwaves, Antennas and Propagation, vol. 8, no. 1, pp. 16-21, 2014.
[34] N. Decarli, D. Dardari, S. Gezici, and A. A. D’Amico, "LOS/NLOS detection for UWB signals: a comparative study using experimental data," in Proceedings of the IEEE 5th International Symposium on Wireless Pervasive Computing 2010 (ISWPC '10), pp. 169-173, IEEE, Modena, Italy, May 2010.

[35] S. Maranò, W. M. Gifford, H. Wymeersch, and M. Z. Win, "NLOS identification and mitigation for localization based on UWB experimental data," IEEE Journal on Selected Areas in Communications, vol. 28, no. 7, pp. 1026-1035, 2010. 



The Scientific World Journal
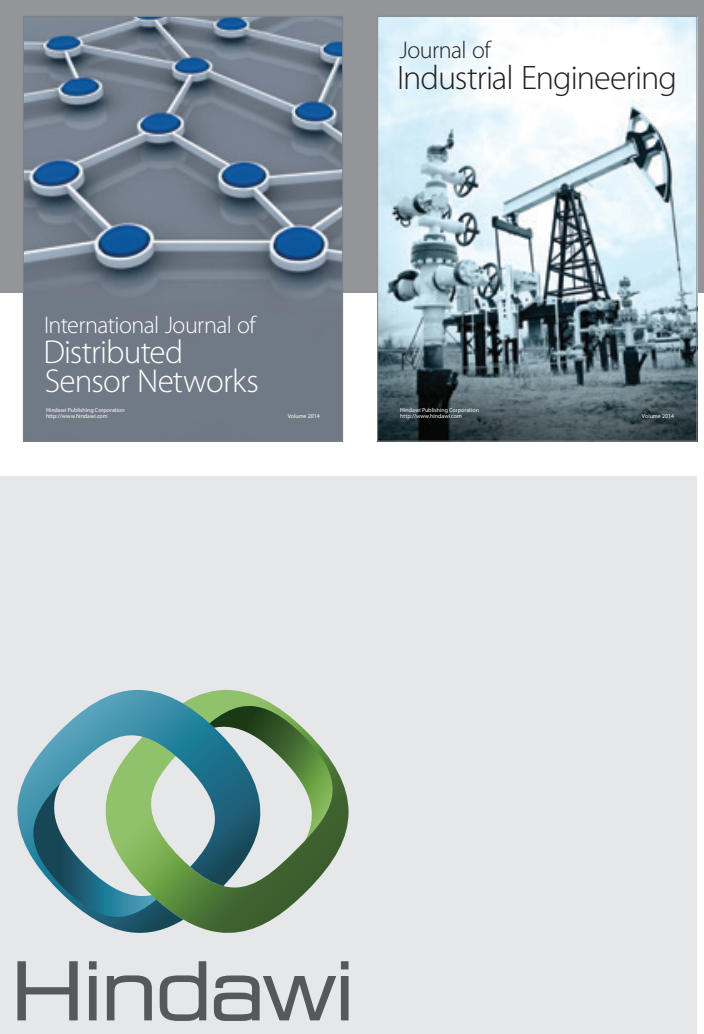

Submit your manuscripts at

https://www.hindawi.com

\section{Computer Networks} and Communications
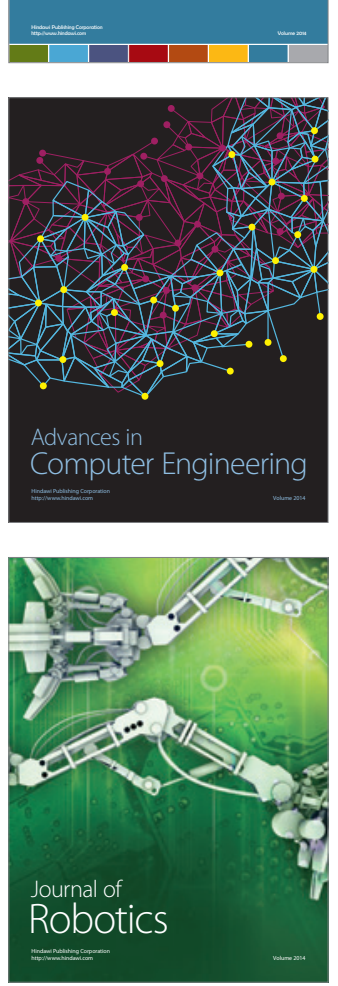
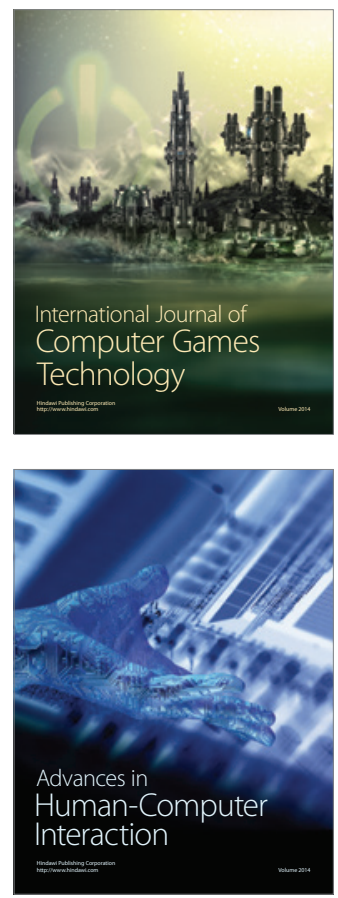
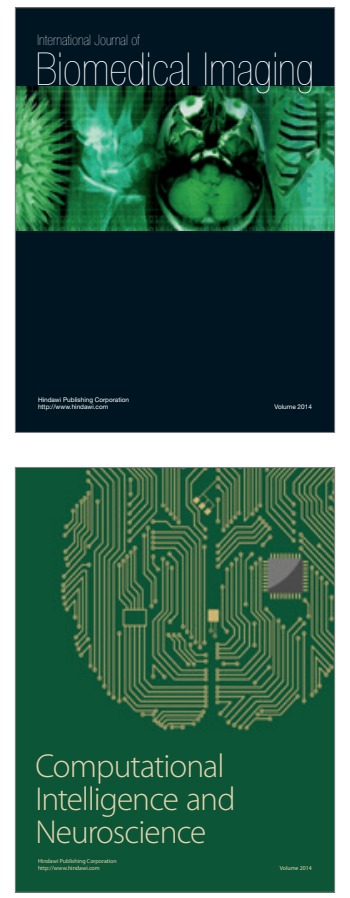
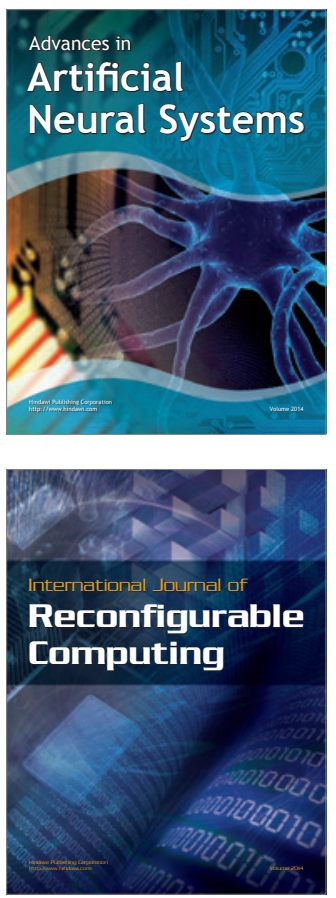
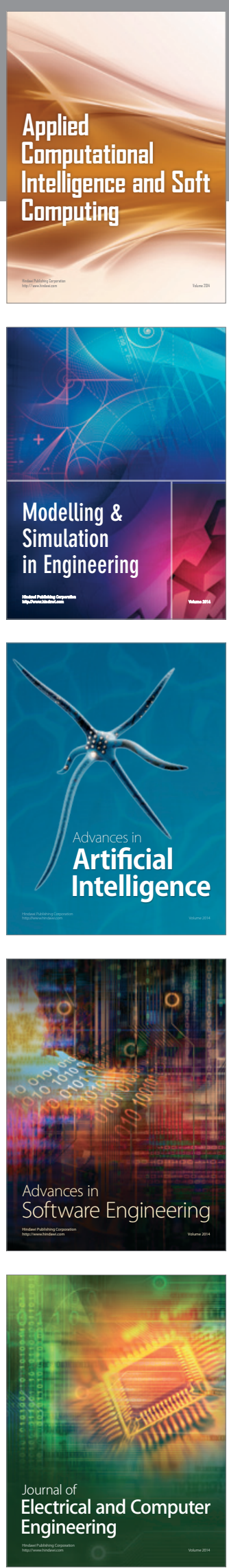